\title{
Myelomatous Pleural Effusion: A Rare Involvement in Myeloma
}

\author{
Onur Alkan a, c, Tarik Onur Tiryaki ${ }^{b}$, Sevgi Kalayoglu-Besisik ${ }^{\mathrm{b}}$
}

\begin{abstract}
Extramedullary disease (EMD) incidence is between $7 \%$ and $18 \%$ in multiple myeloma. Overall survival of patients who develop EMD is significantly shorter than that of patients without EMD. Malignant myelomatous pleural effusions (MPEs) are rarely observed, occurring in less than $1 \%$ of cases. The diagnosis of MPE was confirmed by the detection of myeloma cells in the pleural fluid using flow cytometric analyses. We present a case of a 67-year-old male patient with IgGkappa myelom. After a few line treatment regimens, he was admitted to hospital with back pain and blurred consciousness, and pleural effusion was detected. Pleural fluid analysis showed malignant plasma cells. It is a rare presentation of multiple myeloma, but important in diagnosis.
\end{abstract}

Keywords: Multiple myeloma; Myelomatous pleural effusion; $\mathrm{Cy}$ tometric analysis

\section{Introduction}

Multiple myeloma (MM), although a rare disease, is the second most common hematologic malignancy [1]. In 2019, the number of newly diagnosed myeloma cases is 32,110 in the USA [2]. Differential diagnosis is important due to the frequency of the disease and different forms of presentation. Among the extramedullary involvements of MM, pleural effusion is rare. Although there is no clear consensus on the treatment of myelomatous involvement, the main treatment is MM treatment.

\section{Case Report}

A 67-year-old male patient presented with back pain in Novem-

Manuscript submitted January 29, 2020, accepted February 26, 2020

aDepartment of Internal Medicine, Faculty of Medicine, Istanbul University, Istanbul, Turkey

${ }^{b}$ Division of Hematology, Department of Internal Medicine, Faculty of Medicine, Istanbul University, Istanbul, Turkey

${ }^{\mathrm{c} C}$ Corresponding Author: Onur Alkan, Department of Internal Medicine, Faculty of Medicine, Istanbul University, Istanbul, Turkey.

Email: onur_lkn@hotmail.com

doi: https://doi.org/10.14740/jmc3428 ber 2016. The patient was diagnosed as IgG-kappa MM. Conventional cytogenetic and fluorescence in situ hybridization (FISH) studies $(\mathrm{t}(14 ; 20), \mathrm{t}(6 ; 14), \mathrm{t}(4 ; 14), \mathrm{t}(14 ; 16), \mathrm{t}(11 ; 14), 11 \mathrm{q} 13$, $17 \mathrm{p} 13)$ were done. Conventional cytogenetic analysis showed $46, X Y$ and del(11q13) abnormality was detected. At the time of diagnosis, diffuse bone lesions were noted. The patient underwent two cycles of bortezomib, thalidomide, dexamethasone, cisplatin, etoposide, cyclophosphamide, doxorubicin (VTDPACE) regimen. In April 2017, autologous stem cell transplantation was performed with partial response (PR). Then, loss of biochemical response was observed and lenalidomide treatment was started. In September 2017, very good partial response (VGPR) provided with lenalidomid and second autologous stem cell transplantation was performed. The patient had progression in December 2018 and no response was obtained with two cycles of carfilzomib. Daratumumab treatment was planned.

While daratumumab treatment was planning, in March 2019 , he presented with severe low back pain and blurred consciousness. Shortness of breath developed while being treated. Pleural effusion was detected. Positron emission tomographycomputed tomography (PET-CT) showed bilateral pleural effusion and atelectasis in the lower lobes and widespread bony lesions. Pleural fluid sampling was performed.

Cytospin centrifuge preparation of pleural fluid and cell block sections showed infiltration of malign plasma cells (Fig. 1). CD38 focal positive and CD138 widespread positive were detected in antigenic examinations. CD56 and bcl-1 were negative.

In the follow-up, the patient died due to progressive disease and septic shock.

\section{Discussion}

Extramedullary disease (EMD) incidence is between 7\% and $18 \%$ in MM [3]. Overall survival of patients who develop EMD is significantly shorter than that of patients without EMD. In general, EMD especially plasmocytomas which are not continuous with a bone structure may indicate tumor independence from marrow microenvironment and a gain of invasion and aggressiveness [4]. So, EMD indicates poor prognosis in $\mathrm{MM}$ even in the era of novel agents [4]. Baseline imaging techniques, such as PET scans and magnetic resonance imaging can document whether EMD is present prior to therapy or at progression. As EMD, pleural effusions are rarely associated with MM and most often the result of a concurrent disease process or coexisting illness (e.g. cardiac failure related with amyloidosis or not, chronic renal failure, hypoalbuminemia, 


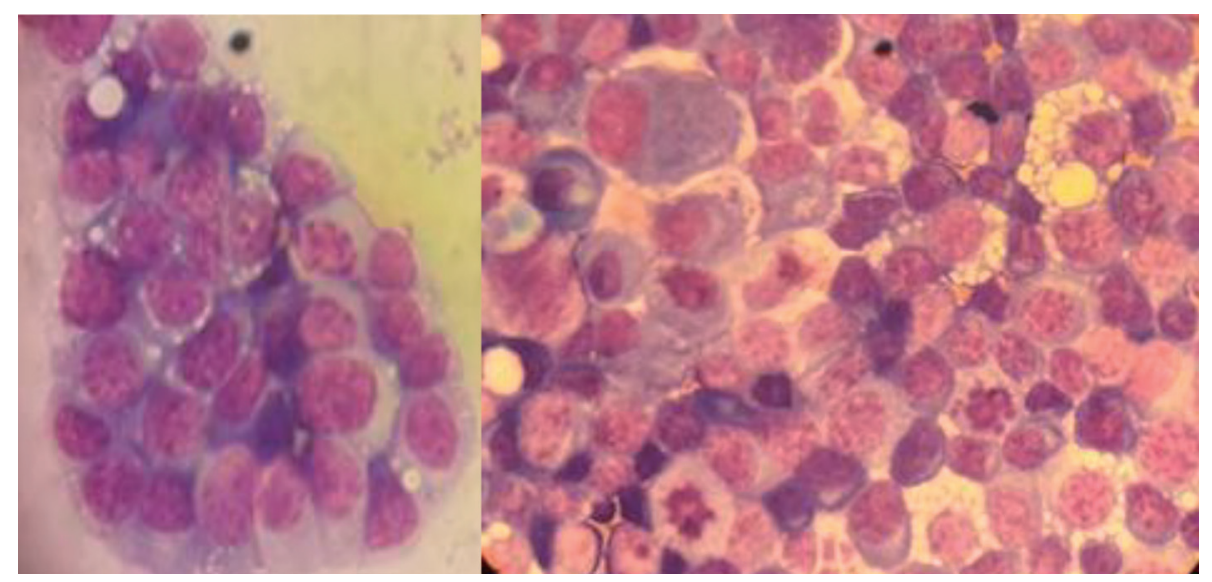

Figure 1. Cytospin centrifuge preparation of pleural fluid revealed atypical plasma cells in different forms.

pulmonary embolism, pneumonia or a second malignancy) [3]. Pleural effusion occurs in approximately $6 \%$ of MM cases [3]. Effusion directly due to myeloma is less than $1 \%$. It is also known to be more common in IgA MM $[3,4]$. Possible causes include invasion from adjacent bone structure, dissemination from plasmocytoma in the chest wall and direct plasma cell infiltration $[5,6]$. It is reported that the prognosis is poor in patients with myeloma causing pleural effusion and it occurs mostly in the late period and survival is reported with a few months [7]. Cytological identification of malignant plasma cells within the pleural effusion has been considered as the best diagnosis method of MPE [6, 8-10]. However, due to the limited number of malignant plasma cells and potential in vitro degeneration, it may fail to make diagnosis.

\section{Acknowledgments}

None to declare.

\section{Financial Disclosure}

This manuscript was not supported by any grant or funding agencies.

\section{Conflict of Interest}

The authors declare that they have no conflict of interest.

\section{Informed Consent}

Informed consent was obtained.

\section{Author Contributions}

OA and TOT wrote the manuscript. SKB edited and provided expertise. All authors have read and approved the manuscript.

\section{Data Availability}

The data supporting the findings of this study are available from the corresponding author upon reasonable request.

\section{References}

1. Kazandjian D. Multiple myeloma epidemiology and survival: A unique malignancy. Semin Oncol. 2016;43(6):676-681.

2. Siegel RL, Miller KD, Jemal A. Cancer statistics, 2019. CA Cancer J Clin. 2019;69(1):7-34.

3. Lang KJ, Lidder S, Aitchison R. Massive pleural effusion due to $\operatorname{IgG}$ multiple myeloma. Hematol Rev. 2009;1(2):e18.

4. Shirdel A, Attaran D, Ghobadi H, Ghiasi T. Myelomatous pleural effusion. Tanaffos. 2007;6:68-72.

5. Gogia A, Agarwal PK, Jain S, Jain KP. Myelomatous pleural effusion. J Assoc Physicians India. 2005;53:734736.

6. Wang Z, Xia G, Lan L, Liu F, Wang Y, Liu B, Ding Y, et al. Pleural Effusion in Multiple Myeloma. Intern Med. 2016;55(4):339-345.

7. Kamble R, Wilson CS, Fassas A, Desikan R, Siegel DS, Tricot G, Anderson P, et al. Malignant pleural effusion of multiple myeloma: prognostic factors and outcome. Leuk Lymphoma. 2005;46(8):1137-1142.

8. Oudart JB, Maquart FX, Semouma O, Lauer M, ArthuisDemoulin P, Ramont L. Pleural effusion in a patient with multiple myeloma. Clin Chem. 2012;58(4):672-674.

9. Keklik M, Sivgin S, Pala C, Eroglu C, Akyol G, Kaynar L, Koker MY, et al. Flow cytometry method as a diagnostic tool for pleural fluid involvement in a patient with multiple myeloma. Mediterr J Hematol Infect Dis. 2012;4(1):e2012063.

10. Magen H. Pleural Effusion in Multiple Myeloma: A Liquid State. Acta Haematol. 2017;138(2):67-68. 